\title{
Quantifying Social Semantics: An Inclusive Definition of Socialness and Ratings for 8,388 English Words
}

\author{
Veronica Diveica $^{1 *}$, Penny M. Pexman ${ }^{2}$, Richard J. Binney ${ }^{1 *}$ \\ ${ }^{1}$ School of Human and Behavioural Sciences, Bangor University, Gwynedd, Wales, UK \\ ${ }^{2}$ Department of Psychology and Hotchkiss Brain Institute, University of Calgary, Canada
}

\begin{abstract}
It has been proposed that social experience plays an important role in the grounding of concepts, and socialness has been proffered as a fundamental organisational principle underpinning semantic representation in the human brain. However, the empirical support for these hypotheses is limited by inconsistencies in the way socialness has been defined and measured. To further advance theory, the field must establish a clearer working definition, and research efforts could be facilitated by the availability of an extensive set of socialness ratings for individual concepts. Therefore, in the current work we employed a novel and inclusive definition to test the extent to which socialness is reliably perceived as a broad construct, and we report socialness norms for over 8,000 English words, including nouns, verbs and adjectives. Our inclusive socialness measure shows good reliability and validity, and our analyses suggest that the socialness ratings capture aspects of word meaning which are distinct to those measured by other pertinent semantic constructs, including concreteness and emotional valence. Finally, in a series of regression analyses, we show for the first time that the socialness of a word's meaning explains unique variance in participant performance on lexical tasks. Our dataset of socialness norms has considerable item overlap with those used both in other lexical/semantic norms and in available behavioural mega-studies. They can help target testable predictions about brain and behaviour derived from multiple representation theories and neurobiological accounts of social semantics.
\end{abstract}

Keywords: word ratings; lexical decision; semantic cognition; social cognition; grounded cognition.

*To whom correspondence may be addressed:

Email:psuda2@bangor.ac.uk/R.Binney@bangor.ac.uk 


\section{Introduction}

Conceptual knowledge is the foundation of our complex interactions with the environment, bringing meaning to the objects, words and social agents we encounter. A major challenge for the cognitive sciences, therefore, is to characterise how meaning is represented in the brain. Of particular interest has been the issue of how the mental representations of concepts become connected to their referents, termed the symbol grounding problem (Harnad, 1990; Searle, 1980). Within multiple representation accounts of semantic processing, concepts are mapped to the world, or grounded, by being directly represented within the neural systems underpinning multiple experiential channels such as perception, action, emotion, language and cognition (Borghi et al., 2018; Kiefer \& Harpaintner, 2020). Sensorimotor systems are particularly important for grounding concrete concepts such as festival and politician. In contrast, abstract concepts like romance and democracy cannot, by definition, be directly experienced through the senses, and may thus rely to a greater degree on other types of information, such as affective (Fingerhut \& Prinz, 2018; Kousta et al., 2011), introspective (Shea, 2018) and linguistic experience (Borghi et al., 2019; Dove, 2018). Further, there is growing recognition that there are different types of abstract concepts which depend to varying extents on these manifold sources of information (Harpaintner et al., 2018; Villani et al., 2019) and which elicit different patterns of behavioural responses in lexical-semantic tasks (Muraki et al., 2020).

Recently, there has been a rise in interest concerning the role that social experience plays in the acquisition and representation of concepts. Indeed, there are proposals in which social interaction and social context are pinpointed as a key source or mechanism for grounding that may be particularly important for the representation of abstract concepts (Barsalou, 2020; Borghi et al., 2019). For instance, Barsalou (2020) proposed that the social environment (e.g., agents, social interaction, culture) provides one form of grounding, in 
addition to that afforded by perceptual modalities, both of which are distinguished from the body, and the physical environment. Likewise, Borghi et al. (2019) argued that both social interactions and linguistic inputs are crucial for the acquisition of abstract concepts (also see Borghi \& Binkofski, 2014). In Pexman, Diveica and Binney (2021), we have reviewed these theoretical perspectives as well as two parallel sets of empirical literature which provide some evidence for socialness being a key principle underpinning semantic representation. For example, property generation and feature ratings studies found that social semantic content, or socialness, helps distinguish concrete from abstract concepts (Barsalou \& WiemerHastings, 2005; Troche et al., 2014; Wiemer-Hastings \& Xu, 2005) and even different subtypes of abstract concepts (Harpaintner et al., 2018; Villani et al., 2019). In parallel, a set of neuroimaging studies have found that words high in socialness are associated with differential patterns of brain activation during semantic processing (e.g., Binney et al., 2016; Mellem et al., 2016; Rice et al., 2018; Wang et al., 2019; for another review, also see Conca et al., 2021). Some authors have argued for a special status of social concepts over other types of concept, and have suggested that socialness may even be a fundamental driver behind the functional organisation of the semantic system (Lin et al., 2018; Ross \& Olson, 2010; Simmons et al., 2010; Zahn et al., 2007). These studies were all based on limited word samples, but they provide some evidence that social words might be a distinct type of concept, in line with proposals of some multimodal (e.g. Borghi et al., 2018) and neurobiological models (e.g., Ross \& Olson, 2010) of conceptual processing.

These theories are nascent and there are many outstanding questions about the nature and extent of the contribution that socialness makes to semantic representation. One fundamental question is whether socialness is a behaviourally relevant principle as indexed, for example, by its ability to account for variance in performance on lexical-semantic tasks. However, the extant empirical support is limited by the way socialness has been defined and 
measured. To our knowledge, the largest source of openly-available socialness norms was compiled by Troche et al. (2017) and includes social interaction ratings for 750 English nouns. Another dataset collected by Binder et al. (2016) includes ratings for 434 nouns, 62 verbs, and 39 adjectives on four socially-relevant dimensions labelled social, communication, human and self. Thus, the scale and scope (i.e., the syntactic classes of words) at which socialness has been explored has been limited to date. Moreover, socialness as a construct has been defined variably in terms of behavioural descriptiveness, and there is no consensus on the criteria that differentiate social from non-social concepts. The heterogeneity in definitions is summarised by Pexman, Diveica and Binney (2021); some researchers have measured socialness as, for example, the degree to which a word's meaning refers to relationships between people (Troche et al., 2014, 2017), to social as opposed to individual contexts (Arioli et al., 2021), or to the relationship between self and others (Crutch et al., 2012), and socialness has also been defined as how well words describe social behaviour (Zahn et al., 2007). This variability in the operationalisation of socialness hinders our ability to compare findings across studies and glean a broader understanding of the contribution made by socialness to conceptual representation in the brain, and its behavioural consequences. Thus, we argue that to further progress theory, the field must first establish a clearer working definition of socialness.

Moreover, many of these past studies employed socialness definitions that emphasise specific aspects of social experience (Pexman et al., 2021). These narrow definitions might neglect important aspects of our highly complex interactions with the social environment. Thus, taking a crucial next step for understanding the construct of socialness, we aimed to collect ratings using an inclusive definition designed to capture all manner of features that are deemed to be socially-relevant. This allowed us to test the extent to which socialness is reliably perceived as a broad construct. Relatedly, our socialness definition can be equally 
applied to a wide range of words, from nouns like those referring to social roles (e.g., lawyer) or institutions (e.g., government), to verbs like to befriend, and adjectives like trustworthy. This broad and inclusive definition can be used as a starting point for future studies exploring more fine-grained aspects of the socialness construct.

In summary, the aims of the present study were as follows: 1) collect socialness ratings for a large set of English words to provide a useful resource for future research endeavours; 2) use an inclusive definition to assess the extent to which socialness is reliably perceived as a broad construct; 3) explore to what extent these new socialness ratings capture aspects of word meaning that are distinct from those measured via other related semantic variables, such as concreteness and emotional valence, and 4) test whether socialness is a behaviourally-relevant construct.

\section{Method}

\section{Participants}

Participants were recruited via the online platform Prolific (https://www.prolific.co/). Responders were restricted to those who self-reported being fluent in English and having no language disorders. A total of 605 participants (359 male, 240 female, 6 unspecified, $M_{\text {age }}=$ 29.44 years, $\left.S D_{\text {age }}=10.6\right)$ completed the study. Participants completed the rating task in 34 minutes on average and were compensated with GBP £4. Following exclusions (see below), the final sample consisted of 539 participants, with ages ranging from 18 to 76 years $(\mathrm{M}=$ $29.7 ; \mathrm{SD}=10.67)$. Of the participants, $216(40.07 \%)$ were female, $317(58.81 \%)$ male and 6 (1.11\%) unspecified. English was the first language for $273(50.65 \%)$ participants. Of the remaining 266 (49.35\%) participants, 111 self-reported as being proficient in English, 124 advanced and 31 beginner/intermediate. A total of 185 (34.32\%) participants were monolingual, while the remaining 354 (65.68\%) reported speaking more than one language. 


\section{Stimuli}

The stimuli were 8,948 words, including 5,569 nouns, 1,343 verbs, 2,009 adjectives, and 26 other part of speech (based on the dominant part-of-speech norms in Brysbaert et al., 2012) ${ }^{2}$. We compiled our stimulus set from two sources: the Calgary Semantic Decision Project (Pexman et al., 2017) and Brysbaert et al. (2014)'s dataset of concreteness ratings. Ratings on emotion dimensions (valence, arousal, dominance) from Warriner et al. (2013) and on concreteness from Brysbaert et al. (2014) are available for all of the words included and the selected words span the entire continuum of these dimensions. In addition, we specifically selected these words so that there would be considerable overlap with behavioural mega-studies and other theoretically important psycholinguistic dimensions, some of which were used in analyses reported below, whereas others might be of interest in future research (e.g., Calgary Semantic Decision Project (Pexman et al., 2017), the Lancaster Sensorimotor Norms (Lynott et al., 2020), the Glasgow norms (Scott et al., 2019), word association norms (De Deyne et al., 2019), word prevalence (Brysbaert et al., 2018)).

We used 30 of the 8,948 words as a set of control items which were to be presented to every participant and used during the data cleaning process (see below). These words were selected based on the ratings received in a pilot study ( $N=36$ participants) that was run to obtain an initial assessment of whether participants understand the task instructions and, in particular, the description of the inclusive socialness measure, and whether they provide reliable ratings (for a detailed description, see Section S1 of Supplementary Materials). Control words were selected to vary in the mean pilot socialness ratings, as well as in their concreteness (Brysbaert et al., 2014) and valence ratings (Warriner et al., 2013).

\footnotetext{
${ }^{2}$ Note that part-of-speech information was not available for one word: hip hop.
} 
In addition to the 8,948 words, we selected 12 practice words to be rated before the main ratings task so that participants could become familiar with the task requirements. We selected practice words that vary in concreteness (Brysbaert et al., 2014) and valence (Warriner et al., 2013), and that span the whole range of the social interaction dimension as measured by Troche et al., (2017) to ensure that participants practised both items with high and with low socialness ratings.

To create the stimulus lists for presentation to participants, we divided the 8,918 words into two lists of 4,459 words equated for letter length, frequency (log subtitle frequency; Brysbaert \& New, 2009), concreteness (Brysbaert et al., 2014) and valence (Warriner et al., 2013). The control words were then added to both lists. Then, we used Qualtrics software (Qualtrics, 2020) to create two questionnaires each with 4,489 words.

\section{Procedure}

The word stimuli were presented using Qualtrics (2020) and linked to the Prolific online recruitment platform (www.prolific.co). Following the consent form, a demographics survey and instructions, participants rated the 12 practice words, then proceeded to rate the main set of items. Each participant rated 370 words randomly selected from one of the two item lists, plus the 30 control words. The control words were randomly intermixed with other items. The full instructions given to participants are presented in Section S2 of supplementary materials. In short, the participants were asked to rate the degree to which the words' meaning has social relevance by describing or referring to the following: a social characteristic of a person or group of people, a social behaviour or interaction, a social role, a social space, a social institution or system, a social value or ideology, or any other socially-relevant concept. 
Participants provided their answers using a 7-point Likert scale presented horizontally below each word. In addition, there was an "I don't know the meaning of this word" option. There were 25 words presented per page. We collected data until we obtained at least 25 ratings per word.

\section{Data cleaning}

In total, we collected 241,575 observations. The data cleaning pipeline involved sequentially implementing several techniques consistent with recommendations for identifying careless or insufficient effort responders (Curran, 2016) and computer-generated random responding (Dupuis et al., 2019), as well as other data cleaning procedures used in previous word norming studies (Brysbaert et al., 2014; Pexman et al., 2019; Warriner et al., 2013). First, we removed data from participants if they completed less than $33 \%$ of the ratings task $(n=0)$, responded with "I don't know the meaning of this word" for more than $25 \%$ of items $(n=8)$ and provided the same rating for more than 25 words in a row $(n=17)$. Next, we examined each participant's ratings of the 30 control words and generated correlations with the mean ratings of those words obtained in the pilot study. We removed data from 36 participants with a correlation coefficient less than .20 . We then computed the correlation between each participant's ratings and the mean ratings of all other participants. We deleted data from 5 participants with a correlation coefficient less than .10. Finally, if more than $15 \%$ of raters reported not knowing a particular word, we removed those words from the analyses reported below. This led to the exclusion of 560 words.

The final dataset was comprised of 8,388 words and 202,841 observations, of which 3,542 were "I don't know the meaning of this word" responses. Not taking into account the control words rated by all participants, each word in the final dataset had 21.92 valid ratings 
on average $(S D=1.68)$, ranging from 15 to 27 ratings. Overall, 7,703 $(91.83 \%)$ words had at least 20 valid ratings.

\section{Data analysis overview}

Data pre-processing, analysis and visualisation was accomplished using RStudio version 3.6.1 (RStudio Team, 2020). We first computed descriptive statistics for the socialness ratings and assessed their reliability. Then, to begin to explore the nature of the information captured by the socialness dimension and characterize its relationship with other pertinent psycholinguistic constructs, we computed the zero-order correlations between the mean socialness ratings and a variety of lexical and semantic properties of the words. Next, we conducted a series of hierarchical regression analyses to examine whether the socialness measure is related to behaviour in lexical tasks, using behavioural responses from the English Lexicon Project (ELP) lexical decision task (LDT; Balota et al., 2007) and the English Crowdsourcing Project (ECP) word knowledge task (Mandera et al., 2020). The LDT outcome variables quantify the speed and accuracy with which participants could distinguish between words and non-word letter strings. The ECP RT outcome variable measures the speed with which participants could recognize a word as known to them, while accuracy is a measure of word prevalence (\% of trials in which a word was identified as known across participants). We selected these tasks because they require only a fairly shallow level of semantic access (Muraki et al., 2020) and thus provide a conservative test of the relationship between this measure and lexical semantic processing. In addition, in both of these tasks, all word stimuli received the same behavioural response ("word" in the ELP LDT, or "I know that word" in the ECP) unlike, for instance, semantic decision tasks (e.g., Pexman et al., 2017) which involve different responses for different types of words. All predictor variables 
were mean-centered and we used reaction times standardized as z-scores because these reduce the influence of individual differences in overall processing speed (Faust et al., 1999).

\section{Results}

\section{Descriptive Statistics}

The resulting socialness ratings are provided on the Open Science Framework (OSF) project page (available at: https://osf.io/2dqnj/). The socialness ratings have a unimodal distribution with a mean of $3.63(S D=1.24)$ (Figure 1A). More descriptive statistics for the mean ratings are provided in Table 1 . The ratings have an average standard deviation of 1.85 $(S D=0.35)$ and participants provided more consistent responses at the extremes of the scale (Figure 1C). Examples of words at the extremes of the socialness dimension are given in Table 2. Words like friendship, people and sociable received high socialness values, while words like avalanche, millimeter and hemoglobin received low socialness ratings, suggesting good face validity.

Table 1. Descriptive Statistics for Socialness Ratings for 8,388 Words.

\begin{tabular}{ll}
\hline Descriptive Statistic & Value \\
\hline Mean & 3.63 \\
Median & 3.57 \\
Standard Deviation & 1.24 \\
Minimum & 1.05 \\
Maximum & 7.00 \\
1st Quartile & 2.62 \\
3rd Quartile & 4.58 \\
Skewness & 0.19 \\
Kurtosis & -0.80 \\
\hline
\end{tabular}


Table 2. List of words at the extremes of the socialness dimension.

\begin{tabular}{llll}
\hline Highest-rated Words & Rating & Lowest-Rated Words & Rating \\
\hline friendship & 7.00 & eucalyptus & 1.05 \\
socialize & 7.00 & horizontal & 1.09 \\
relationship & 6.96 & crocodile & 1.09 \\
people & 6.90 & sulfur & 1.10 \\
romance & 6.78 & sleeve & 1.17 \\
marriage & 6.76 & turbo & 1.18 \\
socialism & 6.75 & cranberry & 1.18 \\
political & 6.73 & dragonfly & 1.18 \\
family & 6.72 & hemoglobin & 1.20 \\
teamwork & 6.72 & shark & 1.21 \\
boyfriend & 6.68 & sunflower & 1.21 \\
friend & 6.68 & sandpaper & 1.22 \\
sociable & 6.68 & millimeter & 1.22 \\
sisterhood & 6.67 & avalanche & 1.22 \\
mother & 6.67 & spinach & 1.22 \\
democracy & 6.65 & airspeed & 1.23 \\
togetherness & 6.65 & button & 1.23 \\
sister & 6.65 & redwood & 1.23 \\
festival & 6.64 & pistachio & 1.24 \\
stepfather & 6.64 & birch & 1.25 \\
humankind & 6.62 & haystack & 1.25 \\
meeting & 6.62 & toothpaste & 1.26 \\
parental & 6.62 & paprika & 1.27 \\
befriend & 6.61 & magnolia & 1.28 \\
chatty & 6.61 & & 1.28 \\
\hline & &
\end{tabular}



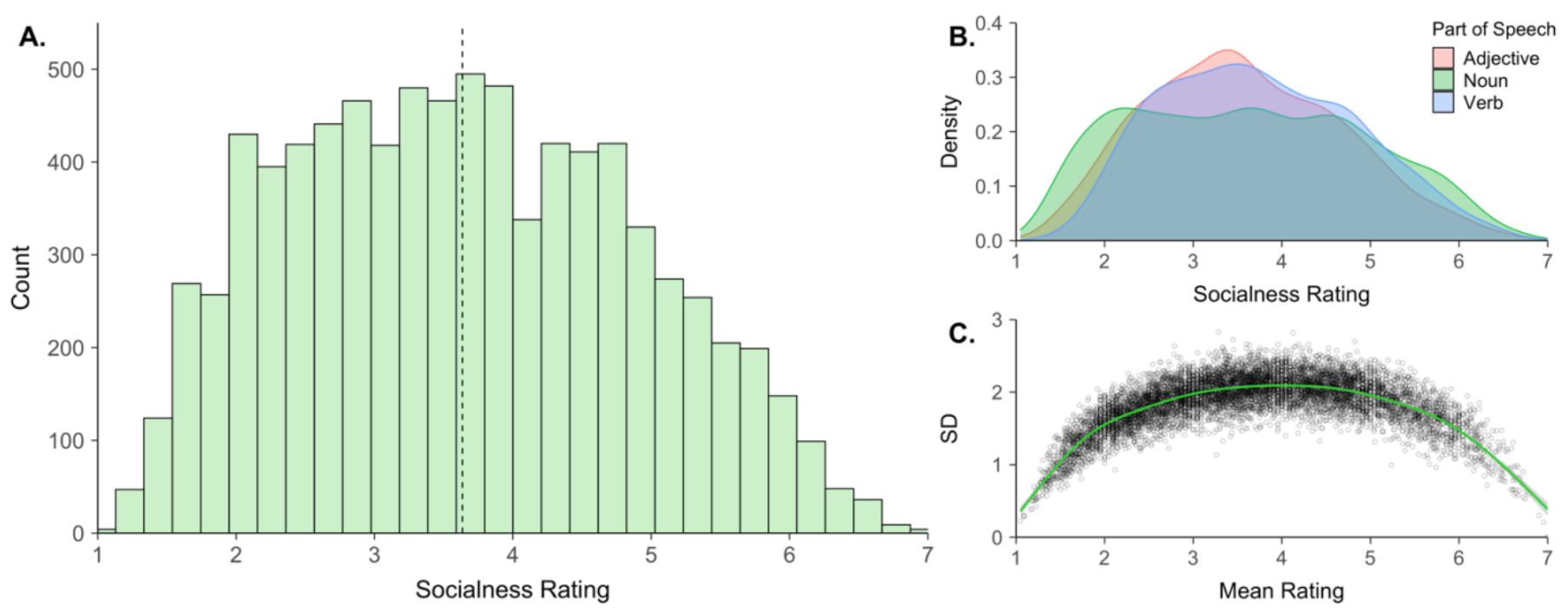

Figure 1. Distribution of socialness ratings. A. Histogram of socialness ratings for 8,388 words; the dotted line represents the mean. B. Kernel density plot of ratings as a function of syntactic class. C. Standard deviation of ratings plotted against their respective mean rating, along with a loess line (in green) that highlights the functional relationship.

\section{Reliability and Validity}

We first examined the reliability of the ratings by computing the one-way intra-class correlation coefficient (ICC) of all ratings using variances estimated via a random effects model with a global intercept and a random intercept per word (Brysbaert, 2019; Stevens \& Brysbaert, 2016). We found an ICC of 0.9 which indicates good reliability of the mean socialness ratings. We further computed the split-half reliability for the 30 control words which were the only items in our dataset rated by all participants. We found a mean Spearman-Brown corrected split-half reliability of $0.998(S D=0.16)$ across 100 random splits, suggesting high reliability for the control items.

We then examined the validity of the ratings by computing the correlations between the ratings observed here and the mean ratings collected in the pilot study ( $n=60$ words), as well as two previous related sets of social interaction norms collected by Binder et al. (2016) 
( $n=258$ words), and Troche et al. (2017) ( $n=450$ words). The current socialness ratings were strongly and positively correlated with the ratings collected in the pilot study $(r=0.97)$ and with the previous social interaction ratings collected by Binder et al. (2016) $(r=0.76)$ and Troche et al. (2017) $(r=0.76)$, suggesting good validity.

\section{Correlations with Lexical and Semantic Properties}

We examined the correlations between the socialness ratings and various lexical and semantic properties of the words. The lexical variables included letter length, orthographic Levenshtein distance (Yarkoni et al., 2008), phonological Levenshtein distance and frequency (log subtitle frequency; Brysbaert \& New, 2009). The semantic variables included concreteness (Brysbaert et al., 2014), imageability (Cortese \& Fugett, 2004; Schock et al., 2012), body-object interaction (BOI; Pexman et al., 2019), sensory experience ratings (Juhasz \& Yap, 2012), valence extremity (i.e. the absolute value of the difference between the valence rating from 5, the neutral point on the scale; Warriner et al., 2013), arousal (Warriner et al., 2013), dominance (Warriner et al., 2013), semantic diversity (Hoffman et al., 2013), rating-based age of acquisition (AoA) (Kuperman et al., 2012), and a test-based AoA measure derived from (Dale \& O’Rourke, 1981) and updated by (Brysbaert \& Biemiller, 2017).

These correlations revealed several interesting relationships that provide insight as to the nature of the word socialness measure (Figure 2; see Figure S1 for scatterplots). Socialness negatively correlated with concreteness $(r=-0.32)$, imageability $(r=-0.18)$, and BOI $(r=-0.17)$, which suggests that words with less social relevance are associated with more embodied sensorimotor information. In contrast, socialness ratings positively correlated with valence extremity $(r=0.22)$ and arousal $(r=0.22)$, suggesting that social words tend to have more affective information. 


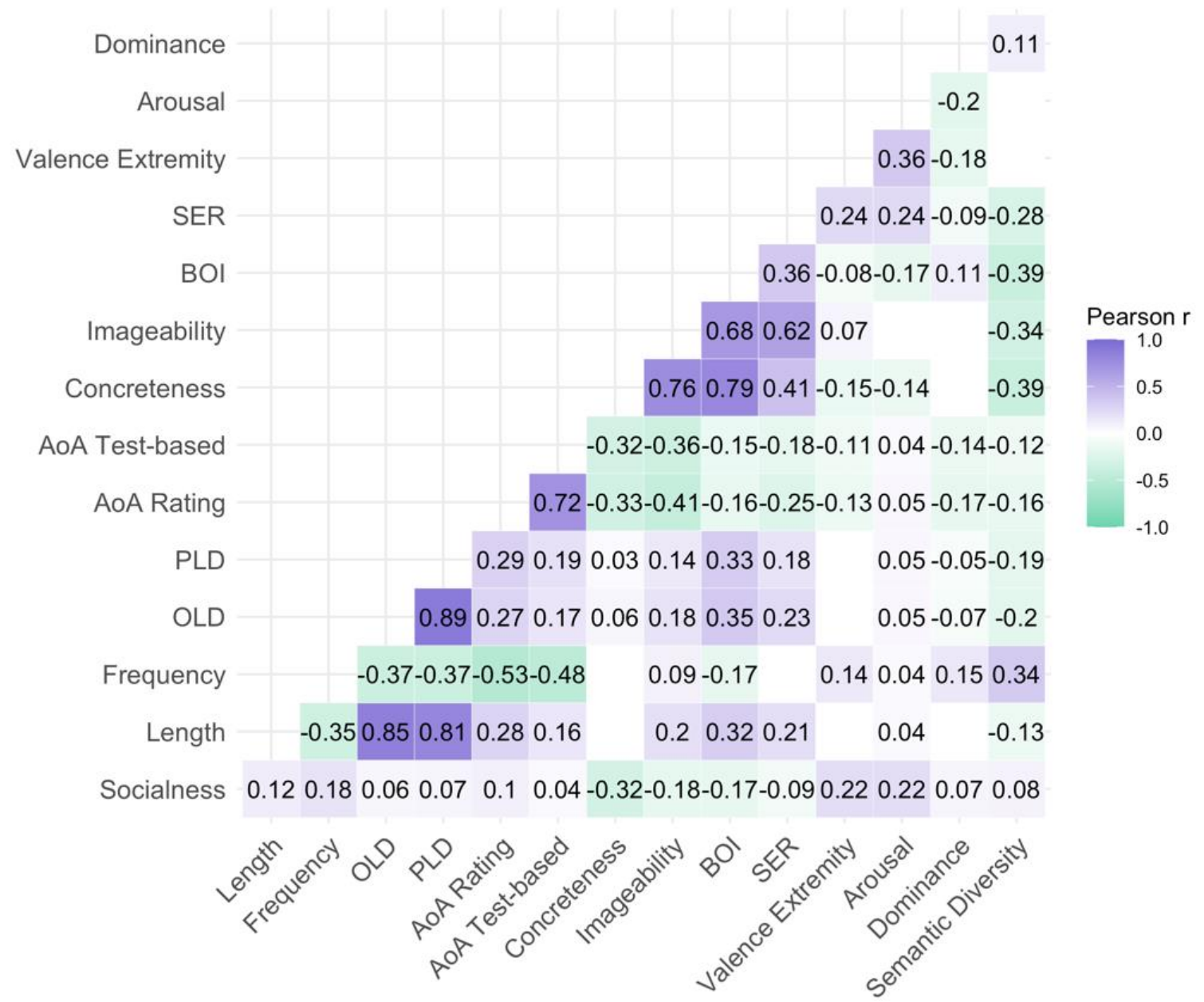

Figure 2. Correlations between mean socialness ratings and lexical-semantic dimensions.

Only correlations significant at $p<.01$ are shown. The strength and direction of the correlation coefficients are indicated by the colour and the numerical values. For each variable of interest, the numbers of items in common with our socialness ratings are as follows: 8,388 for letter length, concreteness, valence, arousal, dominance; 8,160 for frequency; 8,027 for OLD and PLD; 8,348 for rating-based AoA; 7,321 for test-based AoA; 2,680 for imageability; 4,038 for BOI and 2,645 for SER. SER = sensory experience rating; $\mathrm{BOI}=$ body-object interaction; AoA $=$ age of acquisition; $\mathrm{PLD}=$ phonologic Levenshtein distance; OLD = orthographic Levenshtein distance. 


\section{Relationships with Performance on Lexical Tasks}

Next, we examined whether the socialness ratings are related to lexical-semantic processing, using behavioural responses from the ELP LDT (Balota et al., 2007) and the ECP word knowledge task (Mandera et al., 2020). We conducted a series of item-wise hierarchical regression analyses in which we included other lexical and semantic predictors (that are typically related to behaviour in lexical tasks) in order to isolate the unique relationships of socialness to standardized reaction times (RTs) and accuracies. In the first step, we entered the control predictors letter length, frequency (Brysbaert \& New, 2009) and rating-based AoA (Kuperman et al., 2012). In the second step, we entered the semantic predictors: socialness, concreteness (Brysbaert et al., 2014), valence extremity (Warriner et al., 2013) and semantic diversity (Hoffman et al., 2013). We selected these other semantic predictors on the basis of multidimensional theories (e.g., (Borghi et al., 2019) that highlight the simultaneous contribution of semantic variables derived from multiple sources, including linguistic (semantic diversity), sensorimotor (concreteness) and affective experience (valence extremity).

There were 6,926 items for which we had values for all variables of interest in the analysis predicting LDT performance. Descriptive statistics and zero-order correlations between all variables of interest from this dataset are reported in Supplementary Table S1. The statistical results are reported in Table 3 and the standardized coefficients are illustrated in Figure 3A. In this analysis, the control variables were all significant predictors of LDT latencies - RTs were faster for words that are shorter, more frequent and acquired earlier. There was significant improvement in model fit with the addition of the semantic variables, which collectively accounted for a further $0.61 \%$ of variance in LDT latencies. Of the semantic variables, only socialness and semantic diversity were significant predictors, with faster RTs for words with increased social relevance and for those encountered in more 
semantically diverse contexts. A similar pattern of results was observed when predicting LDT accuracy. The control variables were all significant predictors, with better accuracy for words that are longer, more frequent and acquired earlier. There was significant improvement in model fit with the inclusion of the semantic variables, which accounted for an additional $0.56 \%$ of variance in LDT accuracy. Socialness and semantic diversity were the only significant semantic predictors - accuracy was higher for words with increased socialness and for those that are more semantically-diverse. 
Table 3. Regression Coefficients from Item-Level Analyses Predicting Lexical Decision Task Latencies and Accuracy $(N=6,926)$.

\begin{tabular}{|c|c|c|c|c|c|c|c|c|c|c|c|c|c|c|}
\hline \multirow[b]{2}{*}{ Predictor } & \multicolumn{7}{|c|}{ zRTs } & \multicolumn{7}{|c|}{ Accuracy } \\
\hline & $b$ & $S E$ & $t$ & $p$ & $s r^{2}$ & $R^{2}$ & $\Delta R^{2}$ & $b$ & $S E$ & $t$ & $p$ & $s r^{2}$ & $R^{2}$ & $\Delta R^{2}$ \\
\hline Step1 & & & & & & 0.51 & & & & & & & 0.21 & \\
\hline Intercept & -0.25 & 0.003 & -94.49 & $* * *$ & & & & 0.94 & 0.001 & 1049.13 & $* * *$ & & & \\
\hline Length & 0.05 & 0.001 & 35.6 & $* * *$ & 0.09 & & & 0.01 & $<.001$ & 22.57 & $* * *$ & 0.058 & & \\
\hline Frequency & -0.15 & 0.005 & -29.99 & $* * *$ & 0.064 & & & 0.03 & 0.002 & 19 & $* * *$ & 0.041 & & \\
\hline Age of Acquisition & 0.04 & 0.001 & 26.91 & $* * *$ & 0.051 & & & -0.01 & $<.001$ & -22.99 & $* * *$ & 0.06 & & \\
\hline Step2 & & & & & & 0.52 & 0.006 & & & & & & 0.22 & 0.006 \\
\hline Intercept & -0.25 & 0.003 & -95.06 & $* * *$ & & & & 0.94 & 0.001 & 1052.57 & $* * *$ & & & \\
\hline Length & 0.05 & 0.001 & 35.75 & $* * *$ & 0.089 & & & 0.01 & $<.001$ & 21.5 & $* * *$ & 0.052 & & \\
\hline Frequency & -0.13 & 0.005 & -23.9 & $* * *$ & 0.04 & & & 0.03 & 0.002 & 14.7 & $* * *$ & 0.024 & & \\
\hline Age of Acquisition & 0.04 & 0.001 & 25.78 & $* * *$ & 0.046 & & & -0.01 & 0.001 & -22.31 & $* * *$ & 0.056 & & \\
\hline Socialness & -0.01 & 0.002 & -4.73 & $* * *$ & 0.002 & & & 0.003 & 0.001 & 3.57 & $* * *$ & 0.001 & & \\
\hline Concreteness & $<.001$ & 0.004 & 0.02 & 0.984 & 0 & & & -0.002 & 0.001 & -1.7 & 0.088 & 0 & & \\
\hline Valence Extremity & 0.01 & 0.004 & 1.83 & 0.067 & 0 & & & 0.001 & 0.001 & 0.64 & 0.525 & 0 & & \\
\hline Semantic Diversity & -0.07 & 0.01 & -6.77 & $* * *$ & 0.003 & & & 0.01 & 0.003 & 3.54 & $* * *$ & 0.001 & & \\
\hline
\end{tabular}

Note. b represents unstandardized regression weights. SE represents the standard error of the regression weights. sr ${ }^{2}$ represents the semi-partial correlation squared. LDT lexical decision task. zRTs standardized reaction times. ${ }^{*} \mathrm{p}<.05 ;{ }^{* *} \mathrm{p}<.01 ; * * * \mathrm{p}<.001$ 
There were 7,010 items for which we had values for all variables of interest in the analysis predicting performance in the ECP word knowledge task. Descriptive statistics and zero-order correlations between all variables of interest from this dataset are reported in Supplementary Table S2. The statistical results are reported in Table 4 and the standardized coefficients and illustrated in Figure 3B. In this analysis, the control variables were all significant predictors of response latencies - RTs were faster for words that are shorter, more frequent and acquired earlier. There was significant improvement in model fit with the addition of the semantic variables, which accounted for a further $0.78 \%$ of variance in recognition RTs. All semantic variables were significant predictors, with faster RTs for words with increased socialness, concreteness and valence extremity and for those encountered in more semantically diverse contexts. The control variables were all significant predictors of recognition accuracy, with better accuracy for words that are longer, more frequent and acquired earlier. There was significant improvement in model fit with the inclusion of the semantic variables, which accounted for an additional $0.83 \%$ of variance in recognition accuracy. Valence and semantic diversity were the only significant semantic predictors - accuracy was higher for words that are more valenced and encountered in more semantically diverse contexts. 
Table 4. Regression Coefficients from Item-Level Analyses Predicting ECP Word Knowledge Task Latencies and Accuracy (N = 7,010).

\begin{tabular}{|c|c|c|c|c|c|c|c|c|c|c|c|c|c|c|}
\hline \multirow[b]{2}{*}{ Predictor } & \multicolumn{7}{|c|}{ zRTs } & \multicolumn{7}{|c|}{ Accuracy } \\
\hline & $b$ & $S E$ & $t$ & $p$ & $s r^{2}$ & $R^{2}$ & $\Delta R^{2}$ & $b$ & $S E$ & $t$ & $p$ & $s r^{2}$ & $R^{2}$ & $\Delta R^{2}$ \\
\hline Step1 & & & & & & 0.4 & & & & & & & 0.23 & \\
\hline Intercept & -0.53 & 0.001 & -495.33 & $* * *$ & & & & 0.99 & $<.001$ & 5204.69 & $* * *$ & & & \\
\hline Length & 0.01 & 0.001 & 19.37 & $* * *$ & 0.032 & & & 0.002 & $<.001$ & 22.24 & $* * *$ & 0.055 & & \\
\hline Frequency & -0.06 & 0.002 & -27.65 & $* * *$ & 0.065 & & & 0.007 & $<.001$ & 19.99 & $* * *$ & 0.044 & & \\
\hline Age of Acquisition & 0.01 & 0.001 & 25.28 & $* * *$ & 0.054 & & & -0.002 & $<.001$ & -24.02 & $* * *$ & 0.064 & & \\
\hline Step2 & & & & & & 0.41 & 0.008 & & & & & & 0.23 & 0.008 \\
\hline Intercept & -0.53 & 0.001 & -498.44 & $* * *$ & & & & 0.99 & $<.001$ & 5231.36 & $* * *$ & & & \\
\hline Length & 0.01 & 0.001 & 20.21 & $* * *$ & 0.034 & & & 0.002 & $<.001$ & 21.67 & $* * *$ & 0.051 & & \\
\hline Frequency & -0.05 & 0.002 & -22.07 & $* * *$ & 0.041 & & & 0.006 & $<.001$ & 15.74 & $* * *$ & 0.027 & & \\
\hline Age of Acquisition & 0.01 & 0.001 & 22.5 & $* * *$ & 0.043 & & & -0.002 & $<.001$ & -22.38 & $* * *$ & 0.055 & & \\
\hline Socialness & -0.003 & 0.001 & -3.6 & $* * *$ & 0.001 & & & $<.001$ & $<.001$ & 0.31 & 0.754 & 0 & & \\
\hline Concreteness & -0.003 & 0.001 & -2.04 & $*$ & $<.001$ & & & $<.001$ & $<.001$ & -1.46 & 0.145 & 0 & & \\
\hline Valence Extremity & -0.01 & 0.001 & -6.09 & $* * *$ & 0.003 & & & 0.001 & $<.001$ & 3.52 & $* * *$ & 0.001 & & \\
\hline Semantic Diversity & -0.02 & 0.004 & -6.01 & $* * *$ & 0.003 & & & 0.004 & 0.001 & 5.89 & $* * *$ & 0.004 & & \\
\hline
\end{tabular}

Note. b represents unstandardized regression weights. SE represents the standard error of the regression weights. $\mathrm{sr}^{2}$ represents the semi-partial correlation squared. zRTs standardized reaction times. ${ }^{*} \mathrm{p}<.05 ; * * \mathrm{p}<.01 ; * * * \mathrm{p}<.001$ 


\section{A. Lexical Decision Task}

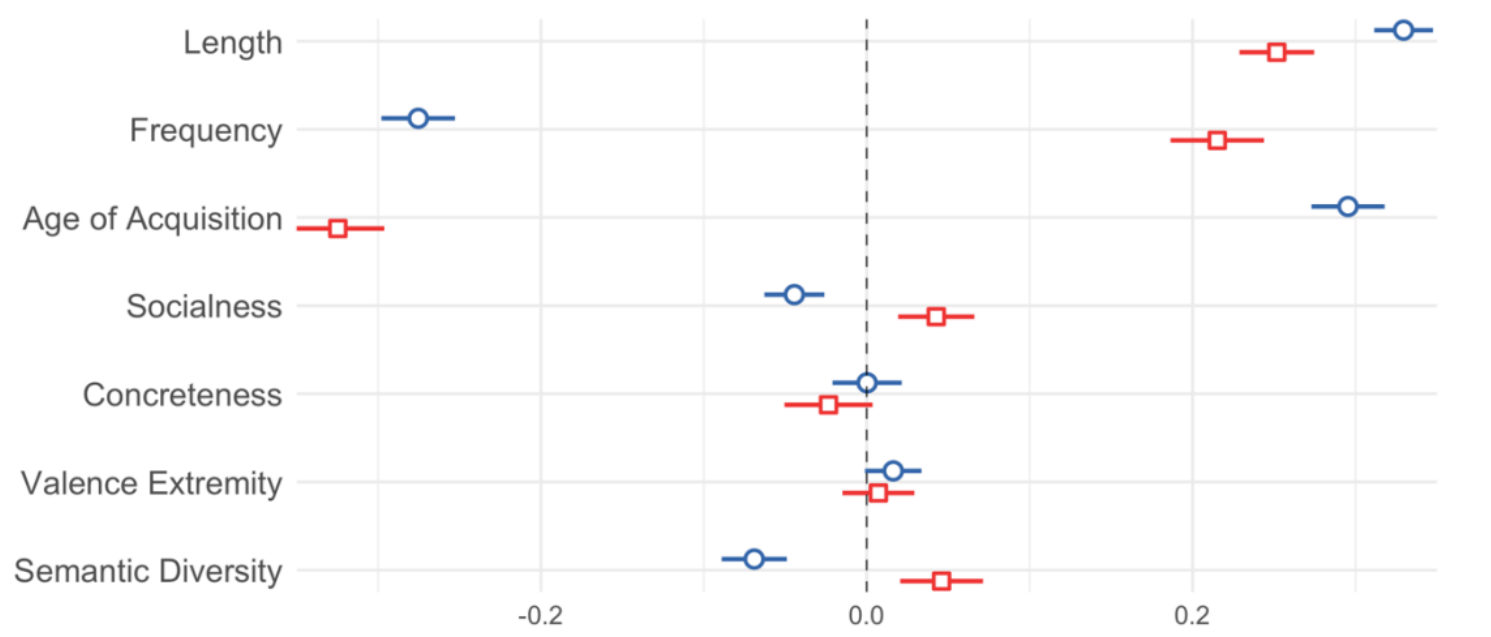

\section{B. Word Knowledge Task}

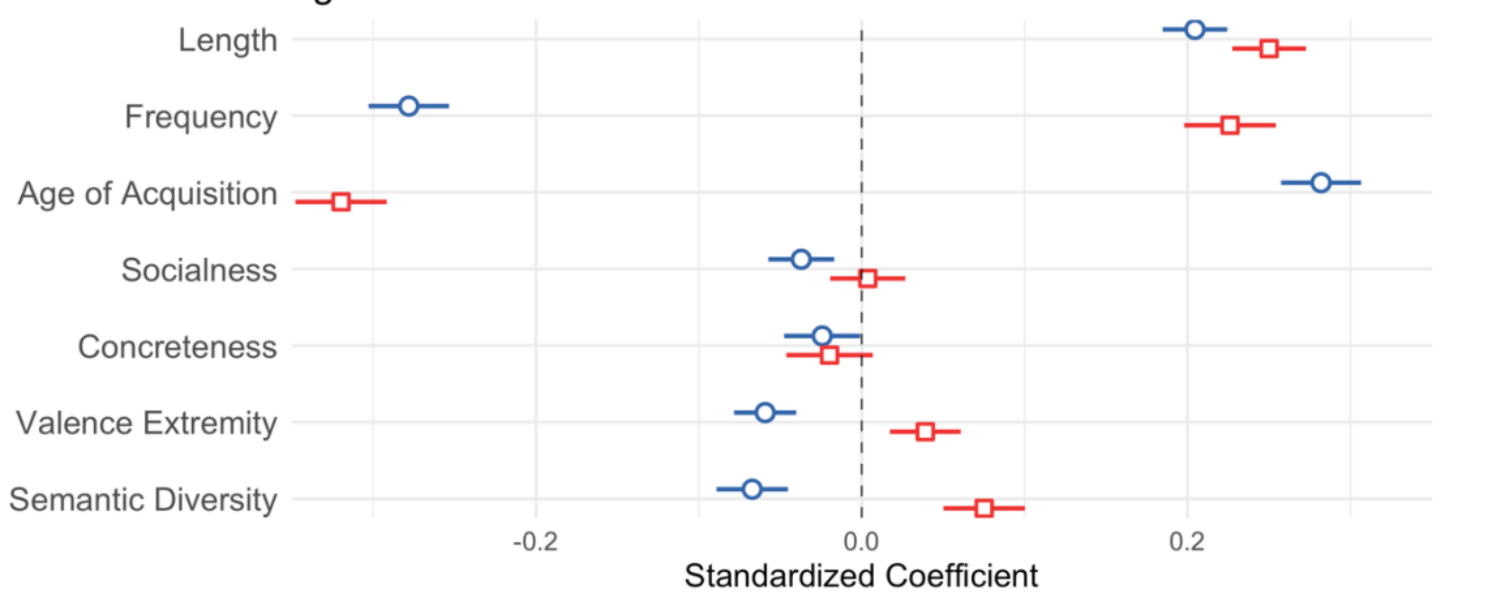

Outcome Variable 0 - Reaction Time $-\square$ - Accuracy

Figure 3. Standardized coefficient weights and 95\%CIs for the second step of the hierarchical regression analyses predicting RTs (blue) and accuracy (red). A. Standardized beta coefficients for LDT outcome variables. B. Standardized beta coefficients for ECP Word Knowledge Task outcome variables. 


\section{Discussion}

Although some contemporary accounts (e.g., Barsalou, 2020; Borghi et al., 2019; Kiefer \& Harpaintner, 2020) proffer a role for socialness in the organization and grounding of conceptual knowledge, many key questions remain about the nature of its contribution and its neural underpinnings. With the aim of facilitating future endeavors, in the present work we sought to 1) collect socialness norms for a large set of words; 2) test the extent to which socialness is reliably perceived as a broad construct; 3 ) explore to what extent socialness captures a distinct aspect of word meaning compared to those measured by other lexical and semantic variables, and 4) assess whether socialness can account for variance in behavioural responses in lexical tasks. To this end, we compiled the largest set of socialness norms available to date by collecting ratings for a set of 8,388 English words, including nouns, verbs and adjectives. The socialness ratings show high reliability, and this suggests that the construct is meaningful to participants even at the broad and inclusive level of description provided. Moreover, the validity of the socialness construct was confirmed by a strong correlation with ratings on two other social semantic dimensions (Binder et al., 2016; Troche et al., 2017), despite the distinct definitions employed. However, our socialness measure shared $57.76 \%$ of variance with either of these other ratings, and this suggests that our more inclusive definition might be capturing some additional aspects of social experience. Subsequent research will be needed to more thoroughly explore the precise aspects of our interactions with the social environment that are captured by this inclusive socialness measure, such as those measured by more restricted definitions (for examples, see Pexman et al., 2021).

However, our preliminary analyses provide some important initial insights into the nature of the socialness dimension. First, while low socialness words tend to be concrete, high socialness words span the entire concreteness continuum, from concrete concepts like 
mother, to more abstract ones like political. In line with previous reports of a negative association between a social interaction measure and modality-specific perceptual ratings (Troche et al., 2017), we found that words high in socialness tend to be more abstract and to rely less on sensorimotor information. However, the present findings further suggest that socialness does not relate to concreteness in a simple linear fashion. Although theories of conceptual representation have proposed that social concepts are a sub-type of abstract concepts (Borghi et al., 2019; Kiefer \& Harpaintner, 2020), this finding highlights the need to better understand the contribution made by socialness beyond this extreme of the concreteness dimension. Second, we found that words with increased socialness tend to be more valenced and arousing. This is in line with findings that social and affective dimensions reduce to the same latent factor of a multidimensional semantic space (Troche et al., 2014, 2017; Villani et al., 2019). Importantly, while the socialness ratings are significantly correlated with all the lexical and semantic variables explored here, the associated effect sizes are modest and suggest that the socialness measure captures a distinct aspect of word meaning. This is consistent with fMRI studies which found that the effect of socialness on brain activation during lexical-semantic processing is independent from that of a number of key semantic variables, namely imageability, concreteness, and valence, and suggest that socialness makes a unique contribution to semantic representation (Lin et al., 2018; Wang et al., 2019).

Using regression analyses, we have demonstrated for the first time that socialness of word meaning is related to performance in lexical tasks. This is true even at the broad and inclusive level of description provided. Specifically, we found a facilitatory effect on behavioural performance in lexical decision and word knowledge tasks - increased socialness was associated with faster decision latencies in both tasks and with better accuracy in the LDT. Importantly, this was true after controlling for other semantic variables known to 
influence lexical-semantic processing, namely concreteness, valence and semantic diversity. Further, this was true even in lexical tasks that involve only shallow semantic processing, where there is a limited pool of variance to be explained by semantic predictors. This unique contribution of the socialness measure suggests that it captures important information about semantic representation and processing. Importantly, our results suggest it contributes to processing alongside other meaning dimensions derived from multiple experiential channels including linguistic (i.e., semantic diversity), sensorimotor (i.e., concreteness) and affective experience (i.e., valence). This is consistent with theories claiming that conceptual representation is multidimensional in nature and that social experience may be one of the underlying semantic dimensions (e.g., Borghi et al., 2019).

However, of note is the observation that the ability of the semantic dimensions to explain variance in behavioural responses varies depending on the requirements of the task. While socialness and semantic diversity had a facilitatory effect on RTs in both tasks, concreteness and valence contributed to the word knowledge task, but not to the LDT. This is in line with research suggesting that conceptual representations are not stable across time and contexts; instead, the aspects of a word's conceptual representation retrieved at any one point depend on the specific task/context (Pexman, 2020; Yee \& Thompson-Schill, 2016). Our pattern of findings may be explained by the fact that LDT only requires the retrieval of some indication that a word has meaning, such as that indexed by its association with a multiplicity of meanings (i.e., semantic diversity). In comparison, the word recognition task might require access to additional features of a word's meaning, like those that tap into the richness of associated sensorimotor (i.e., concreteness) and emotional experience (i.e., valence extremity). It might also suggest that socialness does not contribute additional semantic features to enrich a word's conceptual representation, but is more indicative of the general relevance or salience of its meaning. This might be consistent with our finding that the 
socialness of a word does not account for variance in the number of people who know its meaning. Relatedly, it has been observed that social stimuli are preferentially processed during free viewing of complex naturalistic scenes, to the extent that socialness competes with the physical saliency of stimuli (End \& Gamer, 2017, 2019). However, future research is needed to better understand the nature of the contribution made by socialness to the semantic richness of concepts (see Muraki et al., 2019 for an example of how to approach examining the factor structure of semantic richness).

\section{Conclusion}

In the present study, we compiled the largest set of openly-available socialness norms to date. We used an inclusive definition, found that it produced reliable ratings and, thereby, showed that socialness has meaning as a broad construct. Further, our explorations suggest that socialness captures an aspect of word meaning that is distinct to those measured by other key semantic variables and notably, an aspect of meaning that is behaviourally-relevant. Our study also provides some initial insights into the information captured by the socialness measure, but subsequent work will be needed on this matter, as well as its role and behavioural consequences across the lifespan, including during acquisition, retrieval and when the semantic system is impaired. Thus, the socialness norms described here will enable future research into the organization and grounding of conceptual knowledge, and can help target testable predictions about brain and behaviour that can be derived from multiple representation theories (e.g., Borghi et al., 2019) and neurobiological accounts of social semantics (for an extensive discussion, see Pexman, Diveica and Binney, 2021; also Binney et al., 2016; Binney \& Ramsey, 2020; Diveica et al., 2021). 


\section{Declarations}

\section{Funding}

This work was supported by the Economic and Social Research Council (ESRC) Wales Doctoral Training Partnership in the form of a PhD studentship [ES/P00069X/1], a joint award from UK Research and Innovation (UKRI) and Mitacs under the UK-Canada Globalink Doctoral Exchange Scheme [NE/T014180/1] (both awarded to VD and RJB; PhD student: VD), the Professor Beatrice Edgell Postgraduate Grant from the British Psychological Society Welsh Branch (awarded to VD) and a Social Sciences and Humanities Research Council (SSHRC) of Canada Insight Grant (awarded to PMP).

\section{Competing Interests}

The authors declare no potential conflicts of interest.

\section{Ethics approval}

This study was performed in line with the principles of the Declaration of Helsinki. Ethics approval was granted by Bangor University School of Psychology Ethics Board (Approval Number: 2017-16108).

\section{Consent to participate}

Informed consent was obtained from all individual participants included in the study.

\section{Open Practices and Data/Code Availability Statement}

The datasets generated and/or analyzed in the current study and the analysis scripts are available via the Open Science Framework (OSF) project: https://osf.io/2dqnj/ . Given its exploratory nature, the study was not pre-registered. 


\section{CRediT Author Statement}

Veronica Diveica: Conceptualization, Methodology, Formal Analysis, Investigation, Visualization, Writing - Original Draft, Writing - Review and Editing. Penny Pexman:

Conceptualization, Methodology; Writing - Review and Editing. Richard Binney:

Conceptualization, Methodology; Writing - Review and Editing; Supervision. The authors wish it to be known that PMP and RJB contributed equally to this article.

\section{References}

Arioli, M., Basso, G., Poggi, P., \& Canessa, N. (2021). Fronto-temporal brain activity and connectivity track implicit attention to positive and negative social words in a novel socio-emotional Stroop task. NeuroImage, 226, 117580. https://doi.org/10.1016/j.neuroimage.2020.117580

Balota, D. A., Yap, M. J., Cortese, M. J., Hutchison, K. A., Kessler, B., Loftis, B., Neely, J. H., Nelson, D. L., Simpson, G. B., \& Treiman, R. (2007). The english lexicon project. Behavior Research Methods, 39(3), 445-459. https://doi.org/10.3758/BF03193014

Barsalou, L. W., \& Wiemer-Hastings, K. (2005). Situating Abstract Concepts. In D. Pecher \& R. A. Zwaan (Eds.), Grounding Cognition: The Role of Perception and Action in Memory, Language, and Thinking (pp. 129-164). Cambridge University Press.

Barsalou, Lawrence W. (2020). Challenges and Opportunities for Grounding Cognition. Journal of Cognition, 3(1), 1-24. https://doi.org/10.5334/JOC.116

Binder, J. R., Conant, L. L., Humphries, C. J., Fernandino, L., Simons, S. B., Aguilar, M., \& Desai, R. H. (2016). Toward a brain-based componential semantic representation. Cognitive Neuropsychology, 33(3-4), 130-174. https://doi.org/10.1080/02643294.2016.1147426

Binney, R. J., Hoffman, P., \& Lambon Ralph, M. A. (2016). Mapping the Multiple Graded 
Contributions of the Anterior Temporal Lobe Representational Hub to Abstract and Social Concepts: Evidence from Distortion-corrected fMRI. Cerebral Cortex, 26, 42274241. https://doi.org/10.1093/cercor/bhw260

Binney, R. J., \& Ramsey, R. (2020). Social Semantics: The role of conceptual knowledge and cognitive control in a neurobiological model of the social brain. Neuroscience and Biobehavioral Reviews, 112, 28-38. https://doi.org/10.1016/j.neubiorev.2020.01.030

Borghi, A. M., Barca, L., Binkofski, F., Castelfranchi, C., Pezzulo, G., \& Tummolini, L. (2019). Words as social tools: Language, sociality and inner grounding in abstract concepts. Physics of Life Reviews, 29, 120-153. https://doi.org/10.1016/J.PLREV.2018.12.001

Borghi, A. M., Barca, L., Binkofski, F., \& Tummolini, L. (2018). Varieties of abstract concepts: development, use and representation in the brain. Philosophical Transactions of the Royal Society B: Biological Sciences, 373(1752).

https://doi.org/10.1098/RSTB.2017.0121

Borghi, A. M., \& Binkofski, F. (2014). Words as Social Tools: An Embodied View on Abstract Concepts. Springer. https://doi.org/10.1007/978-1-4614-9539-0

Brysbaert, M. (2019). How Many Participants Do We Have to Include in Properly Powered Experiments? A Tutorial of Power Analysis with Reference Tables. Journal of Cognition, 2(1), 1-38. https://doi.org/10.5334/joc.72

Brysbaert, M., \& Biemiller, A. (2017). Test-based age-of-acquisition norms for 44 thousand English word meanings. Behavior Research Methods, 49(4), 1520-1523. https://doi.org/10.3758/s13428-016-0811-4

Brysbaert, M., Mandera, P., McCormick, S. F., \& Keuleers, E. (2018). Word prevalence norms for 62,000 English lemmas. Behavior Research Methods 2018 51:2, 51(2), 467479. https://doi.org/10.3758/S13428-018-1077-9 
Brysbaert, M., \& New, B. (2009). Moving beyond Kučera and Francis: A critical evaluation of current word frequency norms and the introduction of a new and improved word frequency measure for American English. Behavior Research Methods, 41(4), 977-990. https://doi.org/10.3758/BRM.41.4.977

Brysbaert, M., New, B., \& Keuleers, E. (2012). Adding part-of-speech information to the SUBTLEX-US word frequencies. Behavior Research Methods 2012 44:4, 44(4), 991997. https://doi.org/10.3758/S13428-012-0190-4

Brysbaert, M., Warriner, A. B., \& Kuperman, V. (2014). Concreteness ratings for 40 thousand generally known English word lemmas. Behavior Research Methods, 46(3), 904-911. https://doi.org/10.3758/s13428-013-0403-5

Conca, F., Borsa, V. M., Cappa, S. F., \& Catricalà, E. (2021). The multidimensionality of abstract concepts: A systematic review. Neuroscience \& Biobehavioral Reviews, 127, 474-491. https://doi.org/10.1016/J.NEUBIOREV.2021.05.004

Cortese, M. J., \& Fugett, A. (2004). Imageability ratings for 3,000 monosyllabic words. Behavior Research Methods, Instruments, \& Computers 2004 36:3, 36(3), 384-387. https://doi.org/10.3758/BF03195585

Crutch, S. J., Williams, P., Ridgway, G. R., \& Borgenicht, L. (2012). The role of polarity in antonym and synonym conceptual knowledge: Evidence from stroke aphasia and multidimensional ratings of abstract words. Neuropsychologia, 50(11), 2636-2644. https://doi.org/10.1016/J.NEUROPSYCHOLOGIA.2012.07.015

Curran, P. G. (2016). Methods for the detection of carelessly invalid responses in survey data. Journal of Experimental Social Psychology, 66, 4-19. https://doi.org/10.1016/j.jesp.2015.07.006

Dale, E., \& O'Rourke, J. (1981). The Living Word Vocabulary, the Words We Know: A National Vocabulary Inventory. World Book. 
De Deyne, S., Navarro, D. J., Perfors, A., Brysbaert, M., \& Storms, G. (2019). The “Small World of Words" English word association norms for over 12,000 cue words. Behavior Research Methods, 51(3), 987-1006. https://doi.org/10.3758/s13428-018-1115-7

Diveica, V., Koldewyn, K., \& Binney, R. J. (2021). Establishing a role of the semantic control network in social cognitive processing: A meta-analysis of functional neuroimaging studies. NeuroImage, 245, 118702. https://doi.org/10.1016/J.NEUROIMAGE.2021.118702

Dove, G. (2018). Language as a disruptive technology: abstract concepts, embodiment and the flexible mind. Philosophical Transactions of the Royal Society B: Biological Sciences, 373(1752). https://doi.org/10.1098/RSTB.2017.0135

Dupuis, M., Meier, E., \& Cuneo, F. (2019). Detecting computer-generated random responding in questionnaire-based data: A comparison of seven indices. Behavior Research Methods, 51(5), 2228-2237. https://doi.org/10.3758/s13428-018-1103-y

End, A., \& Gamer, M. (2017). Preferential processing of social features and their interplay with physical saliency in complex naturalistic scenes. Frontiers in Psychology, 8(MAR), 418. https://doi.org/10.3389/FPSYG.2017.00418/BIBTEX

End, A., \& Gamer, M. (2019). Task instructions can accelerate the early preference for social features in naturalistic scenes. Royal Society Open Science, 6(3). https://doi.org/10.1098/RSOS.180596

Faust, M. E., Ferraro, F. R., Balota, D. A., \& Spieler, D. H. (1999). Individual differences in information-processing rate and amount: Implications for group differences in response latency. Psychological Bulletin, 125(6), 777-799. https://doi.org/10.1037/00332909.125.6.777

Fingerhut, J., \& Prinz, J. J. (2018). Grounding evaluative concepts. Philosophical Transactions of the Royal Society B: Biological Sciences, 373(1752), 20170142. 
https://doi.org/10.1098/RSTB.2017.0142

Harnad, S. (1990). The symbol grounding problem. Physica D: Nonlinear Phenomena, 42(13), 335-346. https://doi.org/10.1016/0167-2789(90)90087-6

Harpaintner, M., Trumpp, N. M., \& Kiefer, M. (2018). The Semantic Content of Abstract Concepts: A Property Listing Study of 296 Abstract Words. Frontiers in Psychology, 9, 1748. https://doi.org/10.3389/fpsyg.2018.01748

Hoffman, P., Lambon Ralph, M. A., \& Rogers, T. T. (2013). Semantic diversity: A measure of semantic ambiguity based on variability in the contextual usage of words. Behavior Research Methods, 45(3), 718-730. https://doi.org/10.3758/s13428-012-0278-x

Juhasz, B. J., \& Yap, M. J. (2012). Sensory experience ratings for over 5,000 mono- and disyllabic words. Behavior Research Methods 2012 45:1, 45(1), 160-168. https://doi.org/10.3758/S13428-012-0242-9

Kiefer, M., \& Harpaintner, M. (2020). Varieties of abstract concepts and their grounding in perception or action. Open Psychology, 2(1), 119-137. https://doi.org/10.1515/PSYCH2020-0104

Kousta, S. T., Vigliocco, G., Vinson, D. P., Andrews, M., \& Del Campo, E. (2011). The Representation of Abstract Words: Why Emotion Matters. Journal of Experimental Psychology: General, 140(1), 14-34. https://doi.org/10.1037/a0021446

Kuperman, V., Stadthagen-Gonzalez, H., \& Brysbaert, M. (2012). Age-of-acquisition ratings for 30,000 English words. Behavior Research Methods, 44(4), 978-990. https://doi.org/10.3758/s13428-012-0210-4

Lin, N., Wang, X., Xu, Y., Wang, X., Hua, H., Zhao, Y., \& Li, X. (2018). Fine Subdivisions of the Semantic Network Supporting Social and Sensory-Motor Semantic Processing. Cerebral Cortex, 28(8), 2699-2710. https://doi.org/10.1093/CERCOR/BHX148 Lynott, D., Connell, L., Brysbaert, M., Brand, J., \& Carney, J. (2020). The Lancaster 
Sensorimotor Norms: multidimensional measures of perceptual and action strength for 40,000 English words. Behavior Research Methods, 52, 1271-1291.

https://doi.org/10.3758/s13428-019-01316-z

Mandera, P., Keuleers, E., \& Brysbaert, M. (2020). Recognition times for 62 thousand English words: Data from the English Crowdsourcing Project. Behavior Research Methods, 52(2), 741-760. https://doi.org/10.3758/s13428-019-01272-8

Mellem, M. S., Jasmin, K. M., Peng, C., \& Martin, A. (2016). Sentence processing in anterior superior temporal cortex shows a social-emotional bias. Neuropsychologia, 89, 217224. https://doi.org/10.1016/J.NEUROPSYCHOLOGIA.2016.06.019

Muraki, E. J., Sidhu, D. M., \& Pexman, P. M. (2019). Mapping semantic space: property norms and semantic richness. Cognitive Processing. https://doi.org/10.1007/s10339019-00933-y

Muraki, E. J., Sidhu, D. M., \& Pexman, P. M. (2020). Heterogenous abstract concepts: is “ponder” different from “dissolve”? Psychological Research. https://doi.org/10.1007/s00426-020-01398-X

Pexman, P. (2020). How Does Meaning Come to Mind? Four Broad Principles of Semantic Processing. Canadian Journal of Experimental Psychology. https://doi.org/10.31234/osf.io/scwau

Pexman, P. M., Diveica, V., \& Binney, R. J. (2021). Social Semantics: The Organisation and Grounding of Abstract Concepts. PsyArXiv. https://doi.org/10.31234/OSF.IO/WRBGP Pexman, P. M., Heard, A., Lloyd, E., \& Yap, M. J. (2017). The Calgary semantic decision project: concrete/abstract decision data for 10,000 English words. Behavior Research Methods, 49(2), 407-417. https://doi.org/10.3758/s13428-016-0720-6

Pexman, P. M., Muraki, E., Sidhu, D. M., Siakaluk, P. D., \& Yap, M. J. (2019). Quantifying sensorimotor experience: Body-object interaction ratings for more than 9,000 English 
words. Behavior Research Methods, 51(2), 453-466. https://doi.org/10.3758/s13428$018-1171-\mathrm{Z}$

Qualtrics. (2020). Qaultrics (June, 2021). Qualtrics. https://www.qualtrics.com

Rice, G. E., Hoffman, P., Binney, R. J., \& Lambon Ralph, M. A. (2018). Concrete versus abstract forms of social concept: An fMRI comparison of knowledge about people versus social terms. Philosophical Transactions of the Royal Society B: Biological Sciences, 373(1752), 20170136. https://doi.org/10.1098/rstb.2017.0136

Ross, L. A., \& Olson, I. R. (2010). Social cognition and the anterior temporal lobes. NeuroImage, 49(4), 3452-3462. https://doi.org/10.1016/j.neuroimage.2009.11.012

RStudio Team. (2020). RStudio: Integrated Development for R. RStudio. https://www.rstudio.com/

Schock, J., Cortese, M. J., \& Khanna, M. M. (2012). Imageability estimates for 3,000 disyllabic words. Behavior Research Methods, 44(2), 374-379. https://doi.org/10.3758/s13428-011-0162-0

Scott, G. G., Keitel, A., Becirspahic, M., Yao, B., \& Sereno, S. C. (2019). The Glasgow Norms: Ratings of 5,500 words on nine scales. Behavior Research Methods, 51(3), 1258-1270. https://doi.org/10.3758/s13428-018-1099-3

Searle, J. R. (1980). Minds, brains, and programs. Behavioral and Brain Sciences, 3(3), 417424. https://doi.org/10.1017/S0140525X00005756

Shea, N. (2018). Metacognition and abstract concepts. In Philosophical Transactions of the Royal Society B: Biological Sciences (Vol. 373, Issue 1752). Royal Society Publishing. https://doi.org/10.1098/rstb.2017.0133

Simmons, W. K., Reddish, M., Bellgowan, P. S. F., \& Martin, A. (2010). The Selectivity and Functional Connectivity of the Anterior Temporal Lobes. Cerebral Cortex, 20(4), 813825. https://doi.org/10.1093/CERCOR/BHP149 
Stevens, M., \& Brysbaert, M. (2016). A simple solution for missing observations based on random effects models.

Troche, J., Crutch, S. J., \& Reilly, J. (2017). Defining a conceptual topography of word concreteness: Clustering properties of emotion, sensation, and magnitude among 750 english words. Frontiers in Psychology, 8, 1787.

https://doi.org/10.3389/fpsyg.2017.01787

Troche, J., Crutch, S., \& Reilly, J. (2014). Clustering, hierarchical organization, and the topography of abstract and concrete nouns. Frontiers in Psychology, 5, 360. https://doi.org/10.3389/FPSYG.2014.00360

Villani, C., Lugli, L., Liuzza, M., \& Borghi, A. M. (2019). Varieties of abstract concepts and their multiple dimensions. Language and Cognition, 11(3), 403-430. https://doi.org/10.1017/LANGCOG.2019.23

Wang, X., Wang, B., \& Bi, Y. (2019). Close yet independent: Dissociation of social from valence and abstract semantic dimensions in the left anterior temporal lobe. Human Brain Mapping, 40(16), 4759-4776. https://doi.org/10.1002/HBM.24735

Warriner, A. B., Kuperman, V., \& Brysbaert, M. (2013). Norms of valence, arousal, and dominance for 13,915 English lemmas. Behavior Research Methods, 45(4), 1191-1207. https://doi.org/10.3758/s13428-012-0314-x

Wiemer-Hastings, K. K., \& Xu, X. (2005). Content Differences for Abstract and Concrete Concepts. Cognitive Science, 29(5), 719-736. https://doi.org/10.1207/S15516709COG0000_33

Yarkoni, T., Balota, D., \& Yap, M. (2008). Moving beyond Coltheart's N : A new measure of orthographic similarity. Psychonomic Bulletin \& Review 2008 15:5, 15(5), 971-979. https://doi.org/10.3758/PBR.15.5.971

Yee, E., \& Thompson-Schill, S. L. (2016). Putting concepts into context. Psychonomic 
Bulletin \& Review 2015 23:4, 23(4), 1015-1027. https://doi.org/10.3758/S13423-0150948-7

Zahn, R., Moll, J., Krueger, F., Huey, E. D., Garrido, G., \& Grafman, J. (2007). Social concepts are represented in the superior anterior temporal cortex. Proceedings of the National Academy of Sciences of the United States of America, 104(15), 6430-6435. https://doi.org/10.1073/pnas.0607061104 